\title{
ASO Author Reflection: Adjuvant Chemotherapy After Neoadjuvant Chemotherapy in Pancreatic Adenocarcinoma Does Not Lead to Improved Survival in All Patients
}

\author{
Elizabeth J. Olecki, MD ${ }^{1}$, and Niraj J. Gusani, MD, MS, FACS ${ }^{2}$ (] \\ ${ }^{1}$ Department of Surgery, Penn State College of Medicine, Hershey, PA; ${ }^{2}$ Section of Surgical Oncology, Baptist MD \\ Anderson Cancer Center, Jacksonville, FL
}

\section{PAST}

While landmark trials have established that treatment of early stage pancreatic cancer should include both local and systemic therapy, ${ }^{1,2}$ the ideal sequence of surgery and chemotherapy remains unknown, especially regarding the role of neoadjuvant chemotherapy (NT). Additionally, no high-level evidence currently exists regarding use of adjuvant chemotherapy (AT) after completion of NT and surgical resection, leaving the decision to pursue AT in this growing group of patients to the discretion of individual clinicians without guidance regarding which patients may benefit from additional AT. ${ }^{3}$

\section{PRESENT}

Using the National Cancer Database, this study investigated pathologic subgroups by comparing overall survival in patients who received NT only and those who received NT and AT by stratifying high-risk pathologic traits and lowrisk pathologic traits. ${ }^{4} \mathrm{We}$ found that those with low-risk pathology after NT and surgical resection had a 17-32\% decreased associated mortality with the addition of AT compared with NT alone; however, those with high-risk pathologic traits had no significant survival benefit when comparing those who received NT and those who received NT and AT. These results suggest that the addition of AT after NT in patients with poor prognostic features (high

(C) Society of Surgical Oncology 2021

First Received: 7 January 2021

Accepted: 8 January 2021;

Published Online: 22 February 2021

N. J. Gusani, MD, MS, FACS

e-mail: Niraj.Gusani@bmcjax.com lymph node ratio, high grade histology, and positive surgical margins) subjected these patients to the risks associated with chemotherapy without an observable survival benefit.

\section{FUTURE}

Use of NT in nonmetastatic pancreatic cancer will likely continue to increase, with many ongoing trials investigating NT versus upfront surgical resection. It is important to note that a majority of these trials have routine $\mathrm{AT}$ as a part of their protocols. The results from the current study suggest that the assumption that AT after NT is universally beneficial is likely incorrect and future prospective studies are required to further define which subgroups of patients benefit from AT after NT.

DISCLOSURE The authors declare no conflict of interest.

\section{REFERENCES}

1. Oettle H, Neuhaus P, Hochhaus A, Hartmann JT, Gellert K, Ridwelski K, et al. Adjuvant chemotherapy with gemcitabine and long-term outcomes among patients with resected pancreatic cancer: the CONKO-001 randomized trial. JAMA. 2013;310(14):1473-81.

2. Neoptolemos JP, Dunn JA, Stocken DD, Almond J, Link K, Beger $\mathrm{H}$, et al. Adjuvant chemoradiotherapy and chemotherapy in resectable pancreatic cancer: a randomised controlled trial. Lancet. 2001;358(9293):1576-85.

3. Network NCC. Pancreatic Adenocarcinoma (Version 1.2020) 2019. Available from: https://www.ncen.org/professionals/physic ian_gls/pdf/pancreatic_blocks.pdf.

4. Olecki EJ, Stahl KA, Torres MB, Peng JS, Dixon M, Shen C, et al. Adjuvant chemotherapy after neoadjuvant chemotherapy for pancreatic cancer is associated with improved survival for Patients with low-risk pathology. Ann Surg Oncol. 2021. https://doi.org/10. 1245/s10434-020-09546-8.

Publisher's Note Springer Nature remains neutral with regard to jurisdictional claims in published maps and institutional affiliations. 